\title{
Production Function and Robotization How Can CES Technology Help?*
}

\author{
Françoise Larbre ${ }^{\dagger}$
}

April 27, 2021

\begin{abstract}
Depending on the workers qualification, the use of robots is perceived either as a helpful tool or as a competitor. We analyze the substitution of capital for labor, including the case where the product is entirely made by robots. We use CES production functions and their derived cost functions (the later being surprisingly missing in the literature). We focus on short-run and the case of an elasticity of substitution greater than 1 . We highlight a level of product for which the cost is identical regardless of the factor used. As a joint product, we provide a foundation to cost functions exhibiting first increasing and then decreasing returns to scale (a so far missing justification to the usually assumed shape of cost functions).
\end{abstract}

Keywords: CES production function, elasticity of substitution, cost function, robots JEL Classification: D24, L23

${ }^{*}$ I wish to express my gratitude to Gilbert Abraham-Frois with whom I have had the honor and happiness to share many years within the faculty; I thank him for his support and the numerous highly stimulating conversations we had. I also thank Valérie Mignon, Antoine Rebeyrol and Alain Thomazo for their comments on a first draft of this paper in french (februar 2020).

${ }^{\dagger}$ Paris Nanterre University - flarbre@parisnanterre.fr 


\section{Introduction}

We wish to analyze the substitution of capital for labor, including the case where the product is entirely made by robots, without human work. For this purpose, we adopt a production function that allows one to easily choose the elasticity of substitution between factors of production. We shall focus on constant elasticity of substitution functions (CES) and their associated cost functions (see [Fuss (1987)] for a very interesting review on the duality between production and cost functions).

Performing this work, it appeared that some properties of CES production functions had not been investigated to date. This is particularly striking when we consider the cost functions generated by such production functions. To the best of our knowledge their analytical form has been studied only for the specific case of constant returns to scale (for mathematical developments, the reader is invited to consult [Varian (1992)], one of the few textbooks that is cited in research articles or [Kamien and Schwartz (1968)]). Moreover regarding graphical representations of cost functions generated from CES technologies, they appear to be inexistent whatever the type of returns to scale.

After defining in the general case the long-run cost function associated with a CES production function, we shall fix the value of one of the factor of production (Section 2).

We will then focus on the short-run (Section 3) and expand on the case where the elasticity of substitution is greater than 1 . We show that there exists a production level from which the introduction of robots is necessary. For this level of production, there is, from the point of view of cost, an equivalence between a labor-intensive firm and a firm using robots (without aiming to increase the production, cost cannot be a decision criterion in order to substitute robots for workers). A few cases (Section 4) are proposed for a reflection on the meaning of the parameters of a CES function. We provide a stepby-step introduction to a topic that is not presented in the literature and advocate that the possibilities granted by CES production functions should be explored.

As a joint product, this work provides a foundation to cost functions exhibiting first increasing returns to scale then decreasing returns to scale (a so far missing justification to the usually assumed shape of cost functions).

Notations:

$y$ level of product,

$x_{i} \quad$ amount of factor $i$ used,

$c_{i} \quad$ price of one unit of factor $i /$ user cost of factor $i$,

$C$ total cost,

$V C$ variable cost,

$F \quad$ fixed cost due to a fixed factor,

$D$ quasi-fixed cost,

$\sigma \quad$ elasticity of substitution of the production function 


\section{CES technology and derived cost functions}

[Klump and Preissler (2000)] present a history of CES function. Since its introduction in [Solow (1956)] and its detailed presentation in [Arrow et al. (1961)], it has been widely used to represent technology (and also agent preferences).

Let

$$
\mathcal{F}\left(x_{1}, x_{2}\right)=\left[a x_{1}^{\gamma}+b x_{2}^{\gamma}\right]^{\frac{h}{\gamma}}, \quad a>0, \quad b>0, \quad h>0, \quad \gamma<1
$$

$\mathcal{F}$ is homogeneous of degree $h$, and $\sigma=\frac{1}{1-\gamma}>0$.

The elasticity of substitution is in the range $] 0, \infty[$. This function is thus well suited to study the ease of substitution of a factor of production for another. A possible extreme case is when one factor is not used anymore. This possibility is particularly useful when we want to study the competition between workers and robots.

Factor 1 will represent labor, Factor 2, a.k.a. R2D2, will represent robots. In the last century, on earthwork sites workers were in competition with backhoes. Nowadays, cashiers are in competition with automatic cash registers.

Given a CES technology, given $c_{1}$ and $c_{2}$, what is the minimal cost in order to obtain a given product level? The answer in a long-run optic (assuming that nothing prevents the continuous adjustment of factors of production) is:

$$
C(y)=c_{1} x_{1}^{*}(y)+c_{2} x_{2}^{*}(y)+D \quad \text { or } \quad C(y)=V C(y)+D
$$

where $x_{1}^{*}$ and $x_{2}^{*}$ are the optimal amounts of factors in function of the desired product level,

$$
x_{1}^{*}(y)=y^{\frac{1}{h}}\left[a+b\left(\frac{b}{a} \frac{c_{1}}{c_{2}}\right)^{\frac{\gamma}{1-\gamma}}\right]^{-\frac{1}{\gamma}}
$$

and

$$
x_{2}^{*}(y)=\left(\frac{b}{a} \frac{c_{1}}{c_{2}}\right)^{\frac{1}{1-\gamma}} x_{1}^{*}(y)
$$

We do not detail here the resolution technique, given that it is available in undergraduate textbooks (we very succinctly recall it in Appendix A).

Let us notice that the long-run cost curve associated with a CES production function cannot have the shape of a polynomial of degree 3, which is however the most studied case (the intuition being that production process first have increasing returns to scale before having decreasing returns to scale).

The general shape of the long-run curve depends only on the homogeneity degree of the production function from which it is generated. It is not the case anymore when one of the factors of production is fixed, in a short-run optic. 
Let us assume Factor 2 is fixed at level $\bar{x}_{2}$, the total cost is then (see Appendix A)

$$
C(y)=c_{1}\left(\frac{1}{a}\right)^{\frac{1}{\gamma}}\left(y^{\frac{\gamma}{h}}-b \bar{x}_{2}^{\gamma}\right)^{\frac{1}{\gamma}}+c_{2} \bar{x}_{2}+D
$$

or more concisely:

$$
C(y)=c_{1} x_{1}^{*}(y)+F+D
$$

where $F$ only depends on $\bar{x}_{2}$; if we allow variations in Factor 2 , the envelope of short-run curves is the long-run curve.

\section{Shape of cost curves}

We can consider different values for parameters $h$ and $\gamma$. The graphics of this section are built using a CES production function with increasing returns to scale (please note that this assumption has no influence on the nature of our results about substitution between labor and robots). We assume $D=0$, which means that fixed costs are only due to the non adjustment of a factor of production.

To deal with the question of full replacement of workers by robots, only the case of an elasticity of substitution greater than 1 is relevant. However, for the sake of completeness, and because they are not presented in the literature to the best of our knowledge, we shall also very briefly present short-run cost functions when $\sigma<1$ and $\sigma=1$.

We will observe that according to the value of the elasticity of substitution, the shortrun cost curves have very different shapes.

\subsection{Low elasticity of substitution}

In Figure 1 the long-run curve indeed exhibits increasing returns to scale assumed for the technology. The short-run curves first exhibit increasing returns to scale before decreasing returns to scale. Here, the sequence of returns to scale of different types is induced by the technology, and not a priori assumed as it is often the case. It is an undeniable advantage of the CES technology with low elasticity of substitution (we detail this aspect in Appendix D).

\subsection{Unitary elasticity of substitution}

Here, to avoid numerical graphical problems due to $\sigma \rightarrow 1$, we use the theoretical limit of the production function which is a Cobb-Douglas function. The long-run curve is the envelope of linear short-run curves as we can see in Figure 2. 


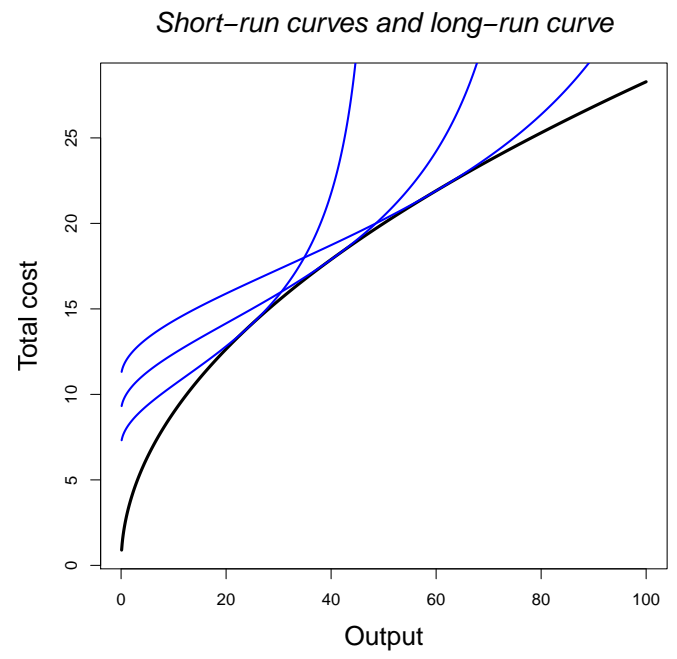

Figure 1: Cost functions in case of low elasticity.

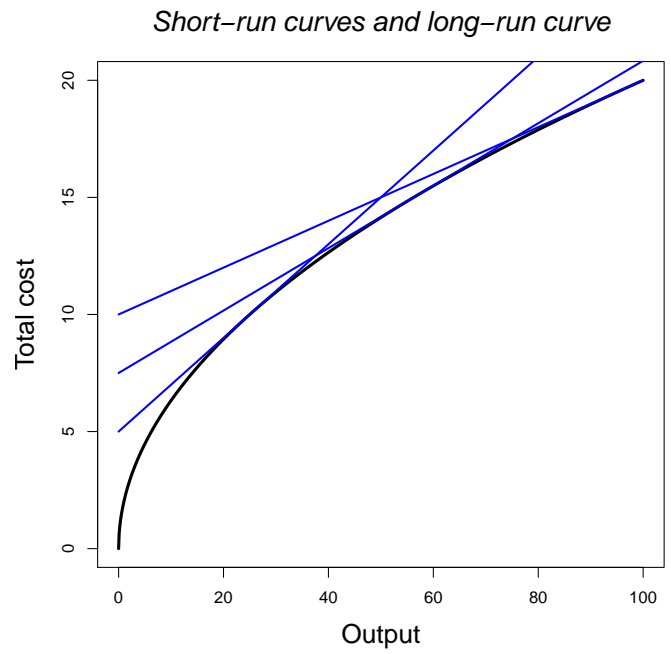

Figure 2: Cost function in case of unitary elasticity.

\subsection{High elasticity of substitution}

We now reach the case of interest for studying the replacement of workers by robots. In the case of an elasticity of substitution greater than 1 , it is possible to produce with only one of the two factors. To draw the curves, one should pay attention to remove the domain that corresponds to a negative quantity of the variable factor. This happens when 
the level of the fixed factor is too high with respect to the level of production (hence an origin of the short-run curves whose abscissa increases with the level of the fixed factor).

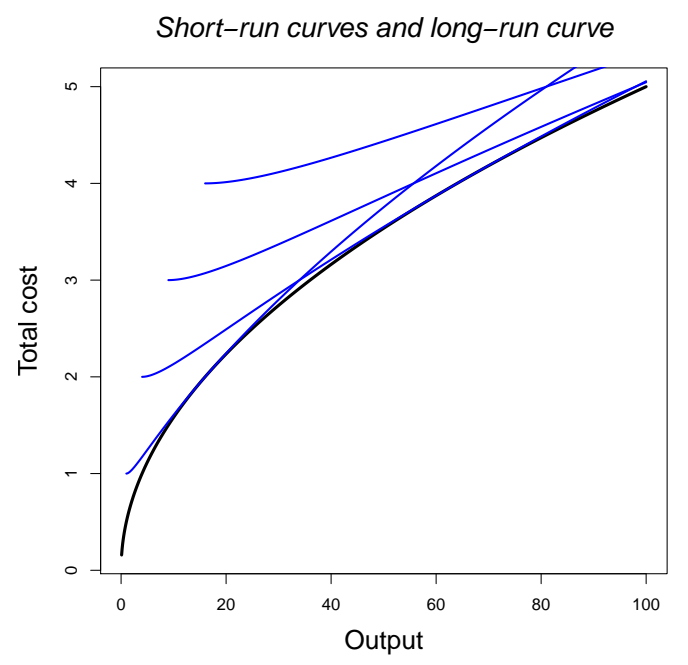

Figure 3: Cost functions in case of high elasticity.

The short-run curves are truncated on the left side, due to negative values for the variable factor, see Figure 3. The lowest point of each short-run curve is associated with $x_{1}=0$, in which case only robots are used (proof in Appendix B).

We want to add on the graphic the short-run curve corresponding to the case $x_{2}=0$, case where robots are not used at all.

We have to distinguish two cases according to the weight of each factor of production:

- case A, both factors of production have the same weight ( $a=b$ and $c_{1}=c_{2}$ ),

- case $\mathrm{B}$, both factors have different weights $\left(a \neq b\right.$ or $\left.c_{1} \neq c_{2}\right)$.

The dashed curve in Figure 4 represents the short-run cost function if robots are not used $\left(\bar{x}_{2}=0\right.$, only labor ensures the production).

When both factors of production have the same weight, along the dashed curve, only labor is used or only robots. Let us note that if $\sigma$ was very high and whatever the value of $x_{2}$, the short-run curves would merge with the long-run curve which means that both factors of production are truly equivalent.

When the factors of production have different weights (due to coefficients in the production function or to their costs), a multitude of situations can be studied. The short-run 

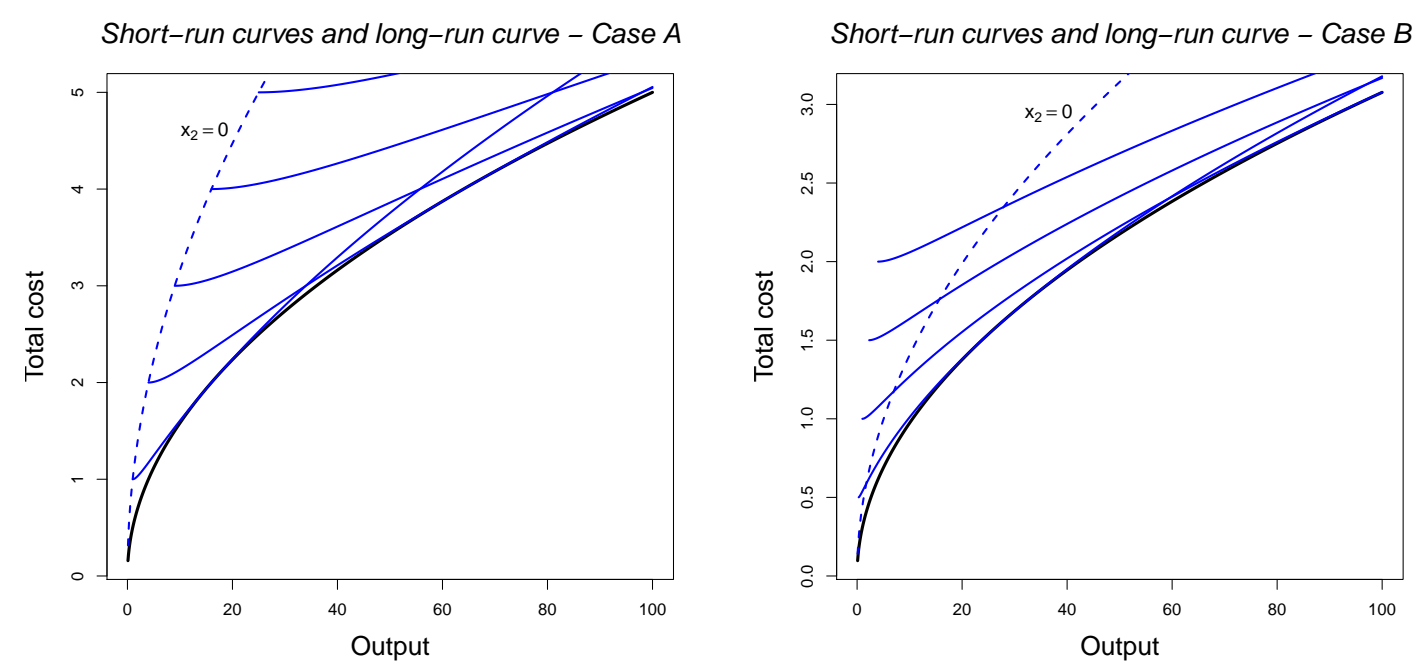

Figure 4: From no robots at all to more or less robots.

cost curve $x_{2}=0$ swings to the right or to the left. For instance, as shown in Figure 4, the dashed curve swings to the right. For a given elasticity of substitution, depending on values of parameters $a$ and $b$, and depending on $\operatorname{costs} c_{1}$ and $c_{2}$, robots will be introduced more or less quickly, then a mix labor-robots is used. An application is proposed in Appendix C.

\section{Human work and robots : a few cases}

Total replacement of a factor by the other one is not eliminated, it will be then supposed that the elasticity of substitution $\sigma$ is greater than $1(\gamma>0)$.

As for examples so far presented, different levels of investment are chosen ; each level defines a short-run cost curve. We choose to show the consequences of a gap between $a$ and $b$ (between $c_{1}$ and $c_{2}$ ) when $c_{1}=c_{2}$ (when $a=b$ ). We also present the case of a very high elasticity.

- Effect of parameters $a$ and $b$ when $c_{1}=c_{2}$, see Figure 5

Quite naturally, when $a<b$ labor is never exclusively used. The alternative only labor or a mix labor-robots appears when $a>b$.

- Effect of costs $c_{1}$ and $c_{2}$, when $a=b$

Graphics would have the same shape than those in Figure 5. When $c_{1}>c_{2}$, labor is never used alone (as when $a<b$ ). The alternative only labor or a mix labor-robots is possible only when $c_{1}<c_{2}$ (as when $a>b$ ). 
Short-run curves and long-run curve with $a<b$

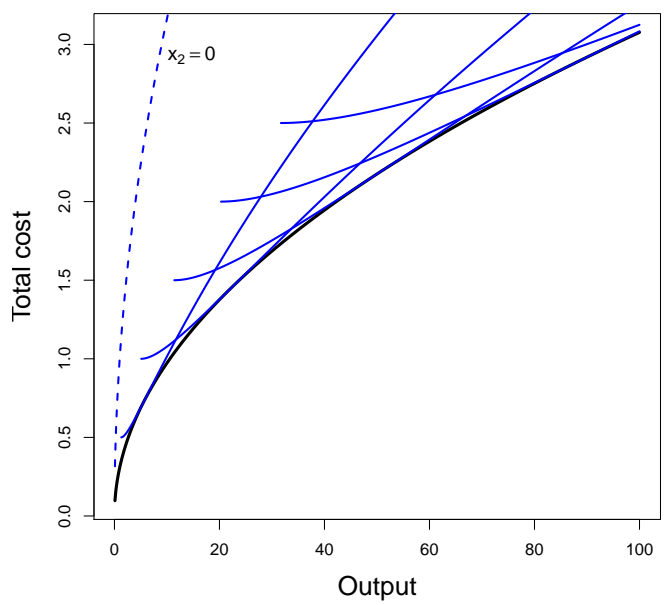

Short-run curves and long-run curve with $a>b$

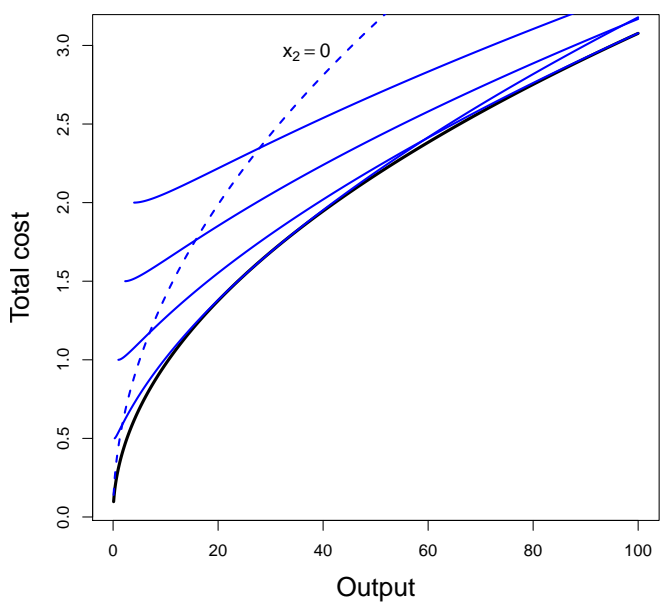

Figure 5: Elasticity $>1$ with $a \neq b$.

- Case of a very high elasticity of substitution, see Figure 6

Factors are perfectly interchangeable.

When $a<b$ (with $c_{1}=c_{2}$ ) or $c_{1}>c_{2}$ (with $a=b$ ), the long-run curve goes through the minimum of short-run cost curves (where $x_{1}=0$ ). Production is carried out entirely by robots.

Short-run curves and long-run curve with $a<b$

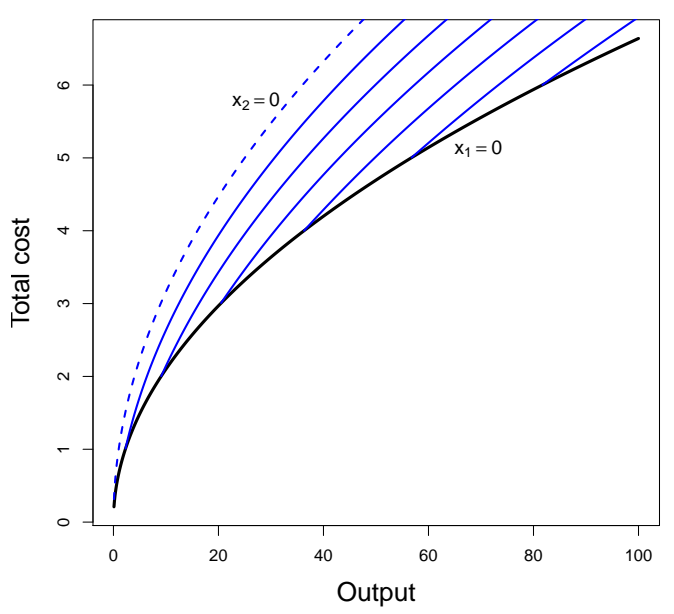

Short-run curves and long-run curve with $a>b$

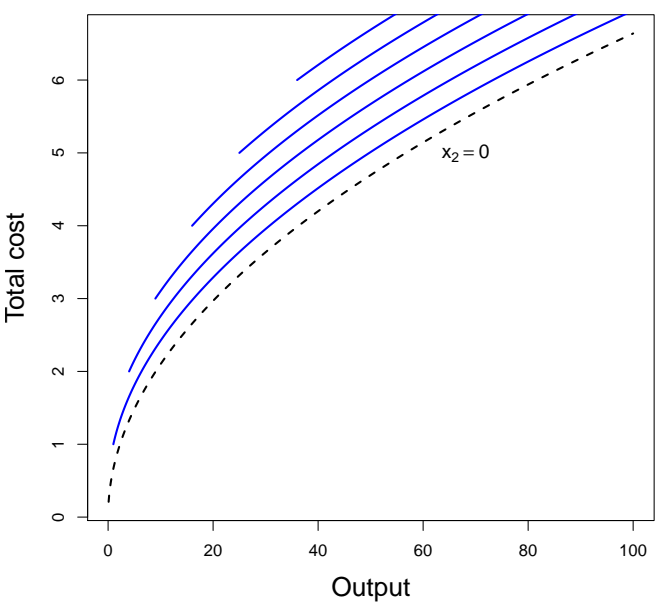

Figure 6: Very high elasticity with $a \neq b$ or $c_{1} \neq c_{2}$. 
When $a>b$ (with $c_{1}=c_{2}$ ) or $c_{2}<c_{1}$ (with $a=b$ ), the curve $x_{2}=0$ merges with the long-run cost curve, robots will never be adopted.

What do $a$ and $b$ mean, or what meaning should they have? Are they just technical parameters or should we consider workers employment, pleasure of hand made things, preference for human presence versus robots?

\section{Concluding Remarks}

The CES production function, as soon as it is not too similar to a Cobb-Douglas function, allows one:

- in the case of a high elasticity of substitution, to show the equivalence with respect to cost, and in the short-run, of the different possible choices between labor and more capitalistic ones, and hence to define the threshold for the introduction of robots,

- in the case of a low elasticity of substitution, to justify the shape of the curves usually assumed (that is, not derived from a prodution function).

The first result is especially important in a setting where the automation of tasks and the introduction of robots is an increasingly obvious threat to the employment of some categories of workers. We can think of an ethical production function. The reflection should be pursued, both at micro and macro levels and could lead to recommendations for economic and social strategy. Work is needed in this direction. This result stems from programming cost functions derived from usual production functions.

The second result, joint product of this study, could help to improve the presentation of cost functions to students. It can also be viewed as an incentive to search for theoretical foundations to long-run cost functions with variable returns to scale.

\section{References}

[Arrow et al. (1961)] Arrow , K.J, Chenery, H.B., Minhas, B.S and Solow, R.M. (1961), "Capital-Labor Substitution and Economic Efficiency," The Review of Economics and Statistics 3 225-250

[Fuss (1987)] Fuss, M.A. (1987). Production and Cost Functions. in Palgrave Macmillan (eds)

[Kamien and Schwartz (1968)] Kamien, M.I. and Schwartz, N.L. (1968). Optimal "induced" technical change. Econometrica. 1 1-17

[Klump and Preissler (2000)] Klump, R. and Preissler, H. (2000). CES Production Functions and Economic Growth. Scandinavian Journal of Economics. 1 41-56 
[Solow (1956)] Solow, R.M.. (1956). A contribution to the Theory of Economic Growth. Quarterly Journal of Economics. 1 65-94

[Varian (1992)] Varian, H.R. (1992). Microeconomic Analysis. 3rd ed. Norton

\section{Appendix}

\section{A. Cost function derived from CES technology}

Given the technology, given the $\operatorname{costs} c_{1}$ and $c_{2}$, what is the cheapest way to get $y$ ?

$$
\mathcal{F}\left(x_{1}, x_{2}\right)=\left[a x_{1}^{\gamma}+b x_{2}^{\gamma}\right]^{\frac{h}{\gamma}}, \quad a>0, \quad b>0, \quad h>0, \quad \gamma<1
$$

Demands for factors have to be such that marginal rate of technical substitution is equal to the factor cost ratio.

$$
\begin{gathered}
\frac{\mathcal{F}_{x_{1}}}{\mathcal{F}_{x_{2}}}=\frac{c_{1}}{c_{2}} \\
\mathcal{F}_{x_{1}}=\frac{h}{\gamma}\left[a x_{1}^{\gamma}+b x_{2}^{\gamma}\right]^{\frac{h}{\gamma}-1} \gamma a x_{1}^{\gamma-1}
\end{gathered}
$$

and

$$
\mathcal{F}_{x_{2}}=\frac{h}{\gamma}\left[a x_{1}^{\gamma}+b x_{2}^{\gamma}\right]^{\frac{h}{\gamma}-1} \gamma b x_{2}^{\gamma-1}
$$

So we have

$$
\frac{a}{b}\left(\frac{x_{2}}{x_{1}}\right)^{1-\gamma}=\frac{c_{1}}{c_{2}}
$$

implying

$$
x_{2}=\left(\frac{b}{a} \frac{c_{1}}{c_{2}}\right)^{\frac{1}{1-\gamma}} x_{1}
$$

By rewriting technical constraint $y=\mathcal{F}\left(x_{1}, x_{2}\right)$ we get

$$
\begin{gathered}
y=\left[a x_{1}^{\gamma}+b\left(\frac{b}{a} \frac{c_{1}}{c_{2}}\right)^{\frac{\gamma}{1-\gamma}} x_{1}^{\gamma}\right]^{\frac{h}{\gamma}} \\
x_{1}^{*}(y)=y^{\frac{1}{h}}\left[a+b\left(\frac{b}{a} \frac{c_{1}}{c_{2}}\right)^{\frac{\gamma}{1-\gamma}}\right]^{-\frac{1}{\gamma}}
\end{gathered}
$$


and

$$
x_{2}^{*}(y)=\left(\frac{b}{a} \frac{c_{1}}{c_{2}}\right)^{\frac{1}{1-\gamma}} x_{1}^{*}(y)
$$

The long run-cost function is then

$$
C(y)=c_{1} x_{1}^{*}(y)+c_{2} x_{2}^{*}(y)+D
$$

Now assuming that Factor 2 does not adjust anymore, $x_{2}=\bar{x}_{2}$

$$
y=\mathcal{F}\left(x_{1}, x_{2}\right)=\left[a x_{1}^{\gamma}+b \bar{x}_{2}^{\gamma}\right]^{\frac{h}{\gamma}}, \quad a>0, \quad b>0, \quad h>0, \quad \gamma<1
$$

and

$$
x_{1}=\left(\frac{1}{a}\right)^{\frac{1}{\gamma}}\left(y^{\frac{\gamma}{h}}-b \bar{x}_{2}^{\gamma}\right)^{\frac{1}{\gamma}}
$$

The short-run cost function is

$$
C(y)=c_{1}\left(\frac{1}{a}\right)^{\frac{1}{\gamma}}\left(y^{\frac{\gamma}{h}}-b \bar{x}_{2}^{\gamma}\right)^{\frac{1}{\gamma}}+c_{2} \bar{x}_{2}
$$

\section{B. Truncated short-run cost curves}

In the case of high elasticity, we have to show that, at the lowest point of short-run curves, the variable factor is not used. Without quasi-fixed costs, the short-run function is:

$$
C\left(y, \bar{x}_{2}\right)=c_{1}\left(\frac{1}{a}\right)^{\frac{1}{\gamma}}\left(y^{\frac{\gamma}{h}}-b \bar{x}_{2}^{\gamma}\right)^{\frac{1}{\gamma}}+c_{2} \bar{x}_{2}^{\gamma}
$$

Let us first minimize this function.

$$
\begin{gathered}
\frac{d C}{d y}=\frac{1}{\gamma} c_{1}\left(\frac{1}{a}\right)^{\frac{1}{\gamma}}\left(y^{\frac{\gamma}{h}}-b \bar{x}_{2}^{\gamma}\right)^{\frac{1}{\gamma}-1} \frac{\gamma}{h} y^{\frac{\gamma}{h}-1} \\
\frac{d C}{d y}=0
\end{gathered}
$$

Equation (21) has two roots: $y=0$ and $y=b^{\frac{h}{\gamma}} \bar{x}_{2}^{h}>0$, and by injecting the positive value of $y$ in the cost function, we get the minimal cost:

$$
C\left(y, \bar{x}_{2}\right)=c_{2} \bar{x}_{2}
$$

The total cost is only the cost induced by the fixed factor (the only one being used). 
Moreover, we know that the expression of the optimal level of the variable factor is:

$$
x_{1}=\left(\frac{1}{a}\right)^{\frac{1}{\gamma}}\left(y^{\frac{\gamma}{h}}-b \bar{x}_{2}^{\gamma}\right)^{\frac{1}{\gamma}}
$$

which is easily checked to be equal to 0 whenever $y=b^{\frac{h}{\gamma}} \bar{x}_{2}^{h}$.

In the particular case where $a=b$ and $c_{1}=c_{2}$, the curve $\bar{x}_{2}=0$ intersects the other curves at their minimum (which is such that $x_{1}=0$ ). There is indeed an alternative only labor or only robots in the case of a symmetric treatment of both factors.

\section{An application: setting a new plant}

Let us assume that the same service may be obtained using only human labor or only using robots or a mix, and that using robots may only be possible after a minimal investment (1.5 in our example).

In Figure 7 are represented the short-run cost curves for the two possible values for the fixed factor, $x_{2}=0$ or $x_{2}=1.5$.
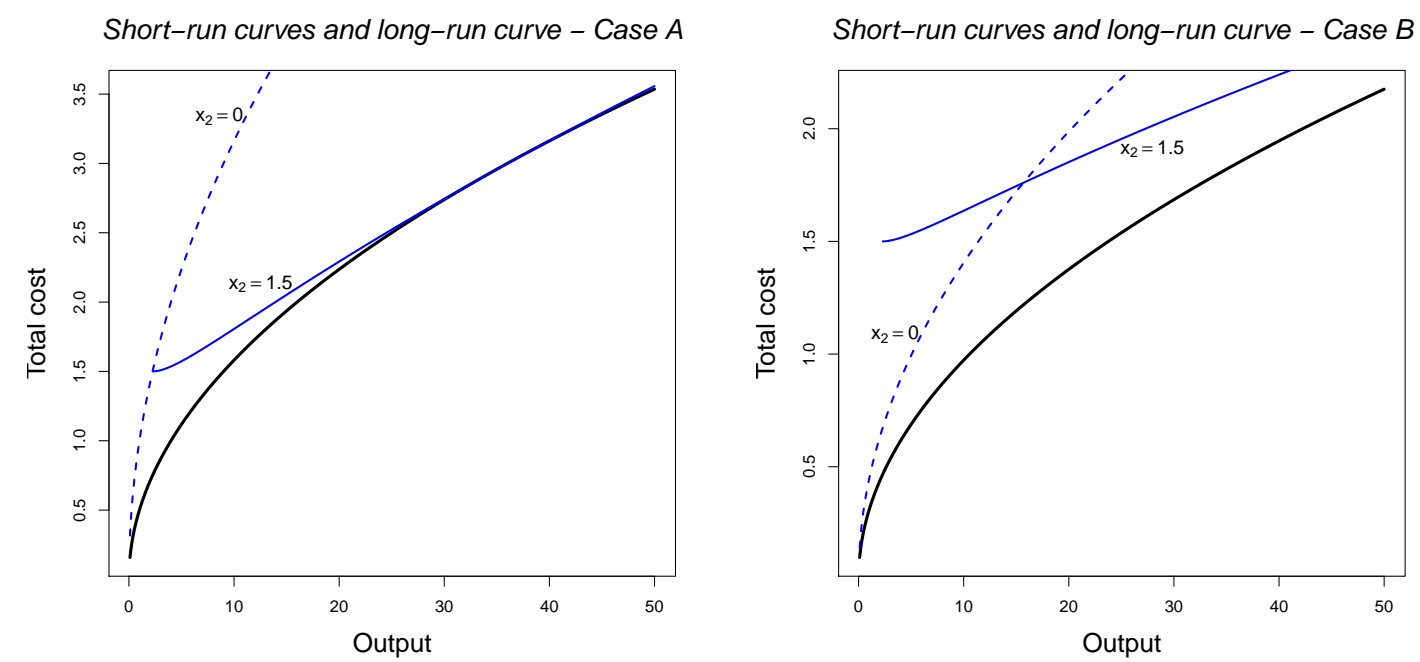

Figure 7: Minimal required investment.

Case A, when both factors of production are weighted similarly, there exists a production level for which it is equivalent, with respect to cost, to use:

- either only labor

- or only robots.

Beyond the intersection point between curve $x_{2}=0$ and curve $x_{2}=1.5$ (only robots or only labor at this point), the combination of labor with robots becomes interesting (with more and more workforce for a given stock of robots). 
Case B, both factors of production are treated in an asymmetric manner. At the intersection of the dashed curve and the plain curve, the alternative is to use:

- either only labor,

- or a combination of labor and robots.

Here again, both alternatives are equivalent with respect to cost, which can thus not be the choice criterion.

It is clear that the meaning of parameters $a$ and $b$ of the production function has to be investigated. The weight given to each factor is clearly decisive for a more or less early introduction of robots. Similarly, the elasticity of substitution may not only be a technical parameter. Should we investigate an ethical production function?

\section{Cost curve with variable returns to scale}

We are now able to give a foundation to usual cost fonctions presented in textbooks, with a decreasing then increasing first derivative. Typically, cost function is often assumed to be of degree 3, but, in this case, it is never derived from an explicit technology.

Supplementary notations:

$A C$ average cost,

$A V C$ average variable cost,

$M C$ marginal cost,

Graphics as in Figure 8 are very common in our textbooks.
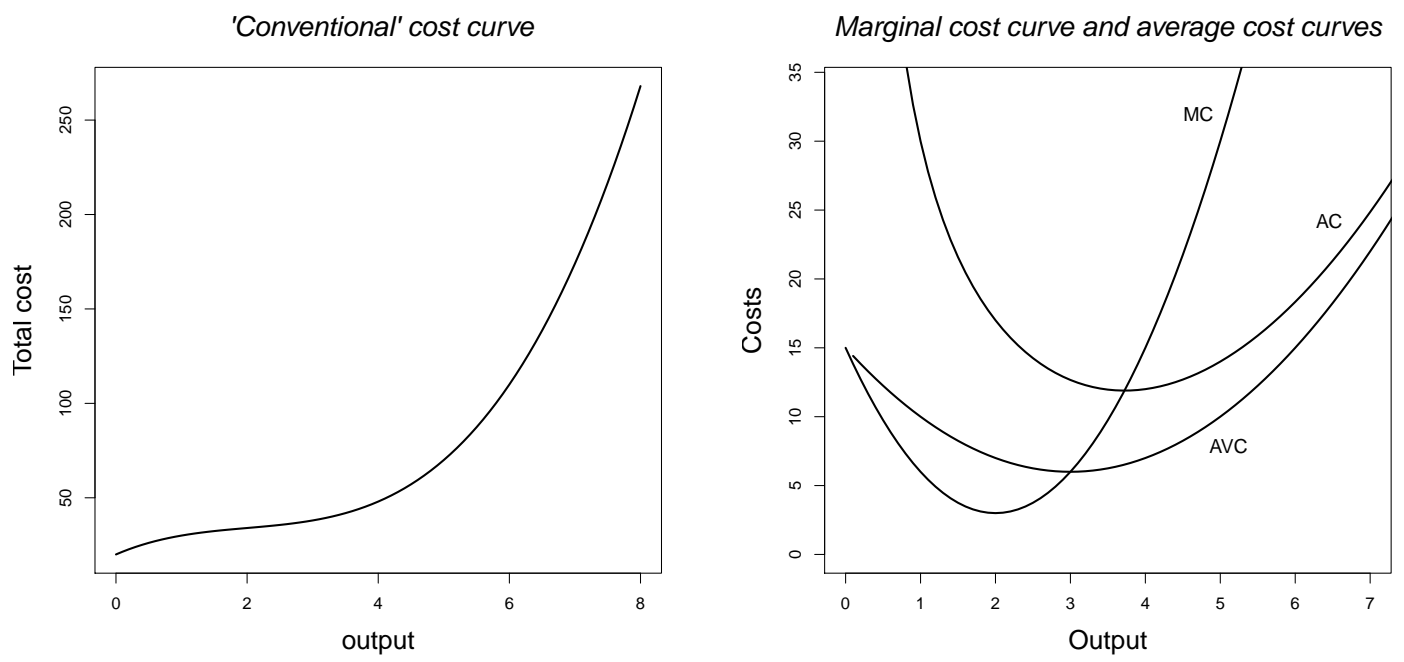

Figure 8: Cost function non derived from a technology. 
The cost function, assumed of degree 3, is such that the marginal cost curve passes through the minimum point of both the average cost curve and the average variable cost curve for $y \neq 0$.

As we know, the long-run cost curve derived from a Cobb-Douglas technology, or in general from a CES technology, does not present such a configuration. The slope of the curve is either always increasing, always constant, or always decreasing, depending on the type of returns to scale. In short-run as well, the shape of cost curves are different from Figure 8 except in the case of low elasticity.

Indeed, the CES functions family, with $\sigma<1$, allows to get graphics such as those in Figure 9.
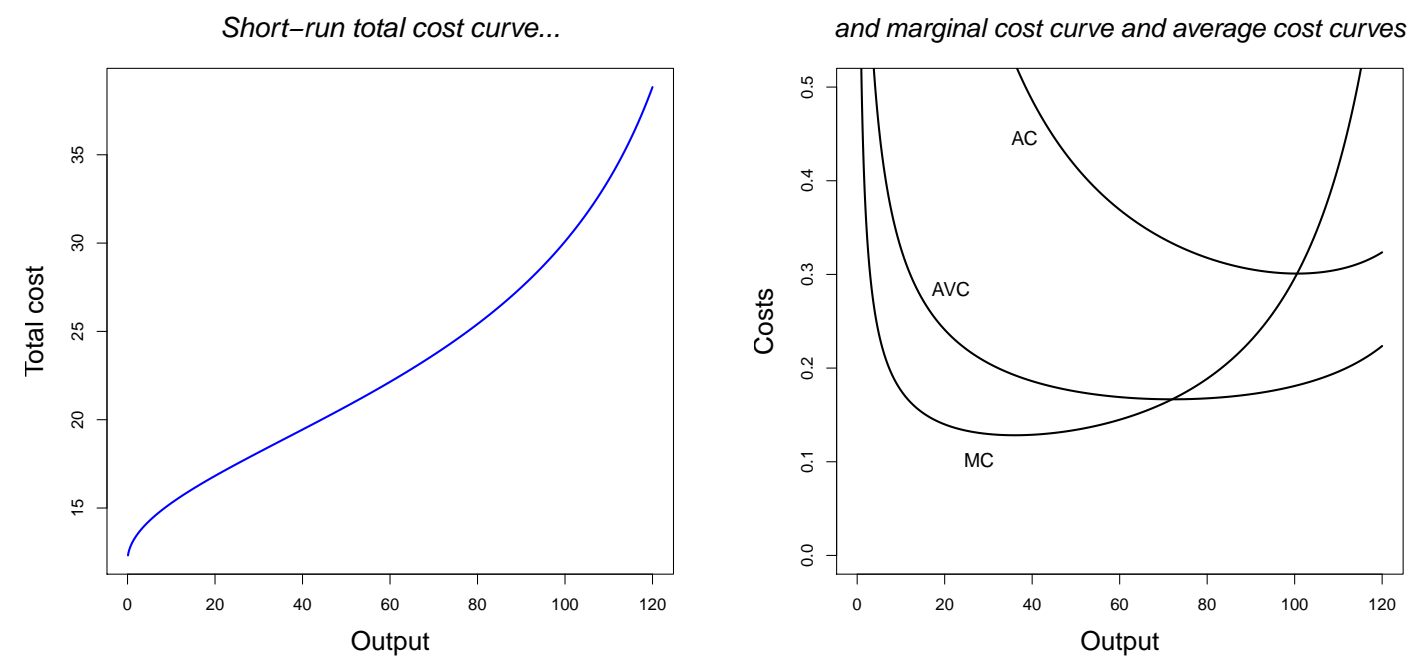

Figure 9: Short-run cost function derived from a CES technology.

The short-run cost curve shows an evolution in returns to scale, first increasing then decreasing. The marginal cost curve and average cost curve have first negative slope then a positive one.

Of course, we can just suppose a cost function of degree 3 , but in this case, we have to give up the graphics exhibiting long-run curves as envelope of short-run curves. The distinction between long term and short term only makes sense if the cost curve is derived from a production function (the long term being defined as the time required to adjust the factors of production). 